\section{In the loop}

Phys. Rev. B (in the press)

Ferrofluids - suspensions of magnetic particles in a fluid - were developed in the 1960 s by NASA scientists who were seeking ways to pump liquid fuels around spacecraft safely, in zero-gravity and without any moving parts. The plan was to drive the ferrofluids with external magnetic fields, to displace and propel other non-magnetic fluids. Unfortunately, to do so required large permanent magnets that were incompatible with the weight constraints of space travel, and to prevent mixing only millimetre-sized fluid channels could be used, limiting flow rates to microlitres per minute.

Using decidedly un-space-age components - including a home-stereo amplifier, PVC piping bought from a local hardware store, and a commercially available ferrofluid of magnetite particles in mineral oil - Leidong Mao and colleagues demonstrate a solution to this problem. The setup consists of a series of solenoid coils wrapped around two sides of a closed loop of pipe, which is filled with ferrofluid. Rather than driving translational motion of a ferrofluid directly with a d.c. field, they apply a phased sequence of oscillating fields that sets its particles rotating. The resulting travelling wave drives the fluid around the loop at rates much faster than had been achieved previously.

\section{Squeezed optics}

Nature Photon. http://dx.doi.org/10.1038/ nphoton.2011.208 (2011)

Mesoscopic optical cavities can alter the emission from any light sources within. Daniel Fuhrmann and colleagues have now shown that acoustic waves can tune the properties of such a cavity while a photon is still trapped inside. This concept could be useful for quantum information processing.

The authors created an optical resonator by perforating a thin membrane of gallium arsenide. An intentional defect in this otherwise regular lattice of holes trapped the light produced by embedded quantum dots. Metal electrodes driven by a radio-frequency voltage generated surface vibrations that travelled across the membrane. These 1.7-GHz sound waves expand and contract the cavity and, in doing so, change its resonant modes. The team observed the changing cavity by monitoring the light emission from the dots.

The real advantage of this approach is its speed: the properties of the photoniccrystal cavity can be altered in a fraction of a nanosecond. This is shorter than both the radiative lifetime of quantum dots and the length of time the photon remains confined in the cavity.

\section{Calorimetric yo-yo \\ Rev. Sci. Instr. 82, 094901 (2011)}

There are a number of parallels between thermal and electric currents. With the design of a thermal analogue of an electrical inductor, Olaf Bossen and Andreas Schilling not only add to the collection, but also enable a new approach to highprecision calorimetry.

Heat capacity can be measured by monitoring the response to an oscillating heating power. One of the advantages of the technique is that the accuracy of a measurement can be improved by temporal averaging. In such experiments, typically the temperature amplitude is monitored and, as the measurement time $t$ is increased, the statistical uncertainty decreases as $t^{-1 / 2}$.

Bossen and Schilling now propose, and demonstrate, an approach in which the heat capacity is related not to the amplitude of the temperature oscillation, but to its frequency. The statistical uncertainty then decreases significantly faster, as $t^{-3 / 2}$. The key to their technique is a construction that neatly combines an electrical conductor, Peltier elements and an amplifier to act as a thermal inductor. When this device is connected to a sample of unknown heat capacity $C$, a resonant circuit can be built that supports autonomous oscillations, at a frequency proportional to $C^{-1 / 2}$.

\section{Plasma screening}

Appl. Phys. Lett. 99, 093701 (2011)

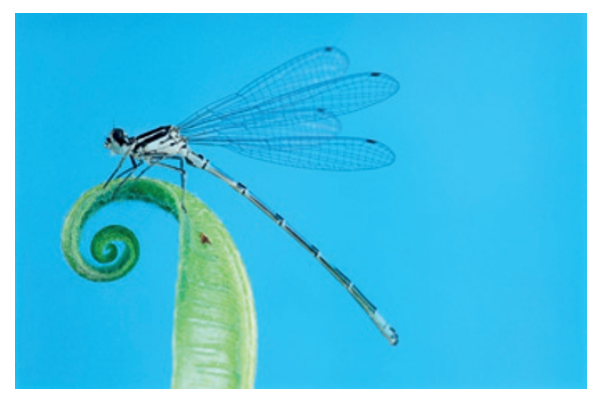

The new generation of laser-driven plasma accelerators has come of age: Stefan Kneip and colleagues have applied their previously reported table-top source for spatially coherent synchrotron X-rays (Nature Phys. 6, 980-983; 2011) to the task of imaging the inner workings of various species of fish and fly.

Although hard tissue such as bone absorbs radiation readily enough to be imaged using conventional X-ray sources, the same technique applied to soft tissue is effective only in conjunction with invasive enhancing agents. Phase-contrast imaging is a more broadly applicable tool, but requires the spatial coherence achieved from a synchrotron.

Kneip et al. have a different solution: by ionizing a milliscale plume of helium gas with a high-power pulsed laser beam, they can set up a plasma wakefield that incites electronic oscillations resulting in the emission of highly spatially coherent synchrotron radiation. For creatures such as the damselfly (pictured), the upshot is that its delicate wing structure can be imaged without using invasive agents. Moreover, the ultrashort pulse duration set by the incident beam means that the technique still works while the damselfly squirms - and hence will have broad implications for research in biomechanics.

Written by Ed Gerstner, David Gevaux, Alison Wright, Andreas Trabesinger and Abigail Klopper 